\title{
ADAPTIVITY IN MC-CDMA SYSTEMS
}

\author{
Ivan Cosovic \\ German Aerospace Center (DLR), Inst. of Communications and Navigation \\ Oberpfaffenhofen, 82234 Wessling, Germany \\ ivan.cosovic@dlr.de \\ Stefan Kaiser \\ DoCoMo Communications Laboratories Europe GmbH \\ Landsberger Strasse 312, 80687 Munich, Germany \\ kaiser@docomolab-euro.com
}

Abstract This paper investigates the performance of MC-CDMA with link adaptation in the uplink of a wireless communication system. Two types of link adaptation approaches can be distinguished: 1) Exploitation of channel state information (CSI) at the transmitter and 2) exploitation of CSI at both the transmitter and the receiver. Based on the theoretical lower bounds for the different approaches we design multi-user concepts and present their performances in fading channels with different system loads. Moreover, real channel estimation is considered in this investigations and the effects of different link adaptation techniques on the peak-to-average power ratio are shown.

\section{Introduction}

Multi-carrier communications realized by orthogonal frequency-division multiplexing (OFDM) have proven to be suitable for broadband wireless communications in several broadcasting and wireless LAN standards [1]. In addition, OFDM is currently under investigation for mobile radio systems beyond $3 \mathrm{G}(\mathrm{B} 3 \mathrm{G})$. In order to further increase the capacity of wireless systems, the exploitation of link adaptation has become an interesting topic of research. Several publications cover link adaptation in orthogonal frequency-division multiple-access (OFDMA) systems, e.g., [2]. In OFDMA bit and power loading can be combined in order to increase the capacity of the system. Compared to OFDMA only few publications cover link adaptation for multi-carrier code-division multiple-access (MC-CDMA), e.g., [3]. With MC-CDMA each data symbol is spread over several subcarriers which can be faded independently. This requires more advanced link adaptation approaches compared to OFDMA. 
In this paper we investigate the application of link adaptation in the uplink of an MC-CDMA system. Different approaches for link adaptation depending on the availability of channel state information (CSI) in the system are considered. When CSI is only available at the transmitter, the link adaptation is based on pre-equalization using power loading. If CSI is available at the transmitter and the receiver, the scheme is referred to as combined-equalization with link adaptation using power loading. These two link adaptation approaches differ significantly as the latter offers more degrees of freedom in the system design than the former. As reference, we include the performance of systems which have CSI available only at the receiver, i.e., post-equalization without link adaptation. The corresponding theoretical lower bounds are presented. Based on these, the achievable performance under real world conditions like channel fading, channel estimation inaccuracies, and different system loads is shown. Since the uplink is sensitive to high peak-to-average power ratios (PAPRs), the influence of the different combined-equalization techniques on the PAPR is compared.

The remainder of the paper is organized as follows. The basic principles of the investigated adaptivity methods for MC-CDMA are described in Section 2. In Section 3, we describe the considered uplink MC-CDMA signal model. The numerical simulations are provided in Section 4, and conclusions are drawn in Section 5.

\section{Adaptivity in MC-CDMA}

Transmission through the frequency-selective fading channel causes degradations of the transmission signal at the receiver. Considering MC-CDMA transmission, the signal-to-noise ratio (SNR) degrades and multiple-access interference (MAI) occurs. To eliminate or reduce the resulting performance degradations, equalization and detection of the received signal based on the CSI estimation can be performed at the receiver.

Alternatively, as illustrated in Fig. 1, CSI can be exploited prior to transmission, i.e., at the MC-CDMA transmitter, as inherently available in, for example, TDD and closed-loop systems. In these systems, the link adaptation can be performed in the form of pre-equalization such that SNR is maximized, MAI is eliminated, or a certain trade-off between these two is achieved. However, when SNR is maximized MAI is further enhanced and, vice-versa, when MAI is eliminated SNR is further degraded. Thus, it can be expected that, if a system is heavily loaded, a technique which compromises these two counteracting effects will not be capable to closely approach the corresponding single-user bounds. 
Adaptivity in MC-CDMA Systems

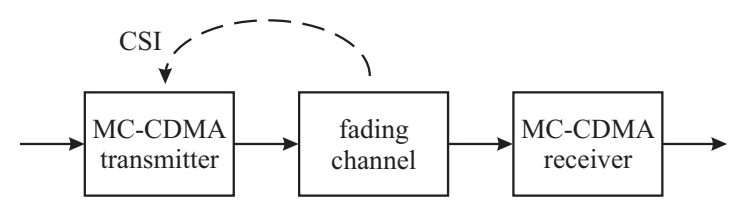

Figure 1. Block diagram of the MC-CDMA transmission system with CSI available only at the MC-CDMA transmitter.

As illustrated in Fig. 2, adaptivity can be used in conjunction with post-equalization at the receiver by exploiting CSI at both MC-CDMA transmitter and receiver. This approach is called combined-equalization and targets a common optimization of data transmission and reception. The main characteristics of combined-equalization are as follows:

- The classical matched filter (MF) bound is valid as the MC-CDMA performance bound for all concepts which are based on pre- and postequalization applied separately. However, this does not hold for combined-equalization Combined-equalization is lower-bounded by the selection diversity (SD) combined-equalization bound that lies well-below the MF bound [4]. The reason for this is based on the fact that, observed from the receiver, the transmission signal is distorted by both link adaptation and fading channel. Hence, detection at the receiver is performed with respect to a pre-equalized fading channel which takes into account both link adaptation and fading channel influence and has different statistics from the fading channel observed alone.

- CSI at both transmitter and receiver ensures more degrees of freedom in the system design and helps to better resolve the problem of joint MAI avoidance/suppression and SNR maximization. In particular, pre-equalization coefficients are designed for efficient distribution of transmit power among the subcarriers with respect to the fading channel, whereas MAI cancellation is the main task of post-equalization. Ideally, the SD combined-equalization bound will be approached closely even if the MC-CDMA system is heavily-loaded.

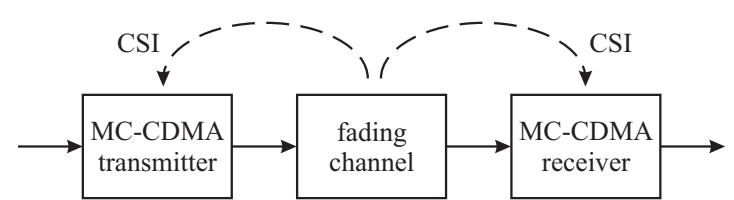

Figure 2. Block diagram of the MC-CDMA transmission system with CSI available at both MC-CDMA transmitter and receiver.

\section{Uplink MC-CDMA Signal Model}

We consider an uplink MC-CDMA system with a total number $N_{\mathrm{c}}$ of subcarriers. The system is divided into $M \cdot Q$ subsystems of $L$ subcar- 
riers. This flexible scheme is better known as MC-CDMA with $M \& Q$ modification [1]. Note, $M$ denotes the number of parallel data symbols transmitted per OFDM symbol for each user, while $Q$ denotes the number of different, parallel user-groups per OFDM symbol. Without loss of generality we concentrate on a single subsystem of $K$ users $(K \leq L)$. The users are separated by spreading sequences of length $L$. Moreover, we assume that the corresponding $L$ subcarriers are spread over the signal bandwidth in order to better exploit frequency diversity of the channel, i.e., they are frequency interleaved with the remaining $N_{\mathrm{c}}-L$ subcarriers. We denote with $l, l=1,2, \ldots, L$, the subcarriers in the considered subsystem. The block diagram of the $k$ th, $k=1,2, \ldots, K$, uplink MC-CDMA transmitter of the considered subsystem is shown in Fig. 3(a). The input bits are channel encoded and symbol mapped producing the complex-valued data symbols. Each data symbol $d^{(k)}$ is spread by a unit-energy spreading sequence $\mathbf{c}^{(k)}=\left(c_{1}^{(k)}, c_{2}^{(k)}, \ldots, c_{L}^{(k)}\right)^{\mathrm{T}}$. The spreading process results in the sequence $\mathbf{s}^{(k)}$ given by

$$
\mathbf{s}^{(k)}=\mathbf{c}^{(k)} d^{(k)}=\left(s_{1}^{(k)}, s_{2}^{(k)}, \ldots, s_{L}^{(k)}\right)^{\mathrm{T}} .
$$

Pre-equalization is applied resulting in a new sequence $\overline{\mathbf{s}}^{(k)}$,

$$
\overline{\mathbf{s}}^{(k)}=\mathbf{G}_{\mathrm{pre}}^{(k)} \mathbf{s}^{(k)}=\left(\bar{s}_{1}^{(k)}, \bar{s}_{2}^{(k)}, \ldots, \bar{s}_{L}^{(k)}\right)^{\mathrm{T}},
$$

where $\mathbf{G}_{\text {pre }}^{(k)}$ is a diagonal $L \times L$ pre-equalization matrix with diagonal elements $G_{\mathrm{pre}, l, l}^{(k)}, l=1,2, \ldots, L$. In this paper, pre-equalization is performed applying generalized pre-equalization (G-pre-eq) whose preequalization coefficients are given by

$$
G_{\mathrm{pre}, l, l}^{(k)}=\left|H_{l, l}^{(k)}\right|^{p} H_{l, l}^{(k) *} \sqrt{\frac{L}{\sum_{n=1}^{L}\left|H_{n, n}^{(k)}\right|^{2 p+2}}}, l=1,2, \ldots, L,
$$

where $H_{l, l}^{(k)}$ represents the fading coefficient on the $l$ th subcarrier for user $k$. G-pre-eq is a unified approach of pre-equalization and comprises several well-known pre-equalization techniques for different values of its design parameter $p$, e.g., pre-equalization maximum ratio transmission (pre-eq MRT) for $p=0$, pre-equalization equal gain transmission (preeq EGT) for $p=-1$, and pre-equalization zero-forcing (pre-eq ZF) for $p=-2$. G-pre-eq is especially suitable for combined equalization since setting $p>-1$ and combining it with post-equalization enables better single-user bounds than the MF bound [4]. However, increasing $p$ is expected to enhance MAI at the receiver as the selectivity of the preequalized fading channel is increased. Hence, as shown in Section 4, 


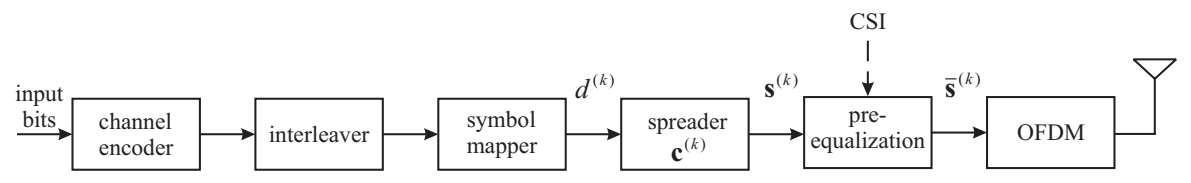

(a)

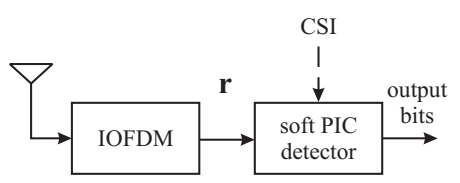

(b)

Figure 3. Block diagram of the uplink MC-CDMA transmission system (a) transmitter and (b) receiver.

the parameter $p$ can be used to achieve a proper trade-off between SNR maximization and MAI elimination in a multi-user uplink MC-CDMA system.

Finally, the pre-equalized sequence $\overline{\mathbf{s}}^{(k)}$ is OFDM-modulated onto the corresponding $L$ out of $N_{\mathrm{c}}$ subcarriers and transmitted. OFDM comprises inverse fast Fourier transform and addition of a guard interval by cyclic extension of the OFDM symbol.

The block diagram of an uplink MC-CDMA receiver of the considered subsystem is shown in Fig. 3(b). After the inverse OFDM (IOFDM) operation the received signal results in

$$
\mathbf{r}=\sum_{k=1}^{K} \mathbf{H}^{(k)} \mathbf{G}_{\mathrm{pre}}^{(k)} \mathbf{s}^{(k)}+\mathbf{n}=\left(r_{1}, r_{2}, \ldots, r_{L}\right)^{\mathrm{T}},
$$

where $\mathbf{H}^{(k)}$ represents the $L \times L$ diagonal channel matrix for user $k$ with the diagonal elements $H_{l, l}^{(k)}, \quad l=1,2, \ldots, L$. The vector $\mathbf{n}=$ $\left(n_{1}, n_{2}, \ldots, n_{L}\right)^{\mathrm{T}}$ represents the AWGN with the one-sided noise spectral density $N_{0}$ in each component. As shown in Fig. 3(b), if CSI is available at the receiver, in this paper, the received signal $\mathbf{r}$ is detected with a soft parallel interference detector (PIC) similar to the one described in [5]. The soft PIC is performed with respect to the pre-equalized fading channel which is defined as

$$
\overline{\mathbf{H}}^{(k)}=\mathbf{H}^{(k)} \mathbf{G}_{\mathrm{pre}}^{(k)} .
$$

Note, if CSI is available only at the transmitter, instead of soft PIC only symbol demapping and channel decoding are performed at the receiver.

\section{Numerical Results}

In this section, several numerical results are given that illustrate the performance of an adaptive uplink MC-CDMA system. 
Table 1. MC-CDMA system parameters for simulations

\begin{tabular}{l|c}
\hline carrier frequency & $5.2 \mathrm{GHz}$ \\
\hline transmission bandwidth & $50 \mathrm{MHz}$ \\
\hline number of subcarriers & $N_{\mathrm{c}}=512$ \\
\hline guard length & $1.8 \mu \mathrm{s}$ \\
\hline OFDM symbols per frame & 64 \\
\hline OFDM frame duration & $0.77 \mathrm{~ms}$ \\
\hline signal constellation & QPSK \\
\hline spreading sequences & Walsh-Hadamard; length $L=8$ \\
\hline system load & full-load $(K=8)$ or half-load $(K=4)$ \\
\hline channel coding & convolutional; rate $R=1 / 2 ;$ memory $m=6$ \\
\hline channel model & ETSI BRAN Model E \\
\hline
\end{tabular}

Table 1 shows the main parameters of the uplink MC-CDMA simulation system. The underlying channel model is based on the Channel $\mathrm{E}$ defined in the ETSI BRAN HIPERLAN/2 standardization project [6]. Unless otherwise stated, we assume that CSI is perfectly known and that mobile speed equals $v=0 \mathrm{~m} / \mathrm{s}$. The SNR is given in $E_{t} / N_{0}$, i.e., the transmitted energy per bit $E_{t}$ over the one-sided noise spectral density $N_{0}$.

First, we consider adaptive uplink MC-CDMA with combined equalization and determine the G-pre-eq parameter $p$ such that the SNR required to achieve an average bit-error-rate (BER) of $P_{\mathrm{b}}=10^{-3}$ is minimized. Fig. 4 shows the required SNR as a function of the parameter $p$. Simulation results are given for fully- $(K=8)$ and half-loaded $(K=4)$ systems, whereas the parameter $p$ of G-pre-eq is varied in order to find the optimum value which minimizes the required SNR. At the receiver soft PIC with 3 iterations is applied. It can be seen that a minimum SNR is achieved by setting $p=1$ and $p \in[4,9]$ in the case of full- and half-load, respectively. The reason for this difference is a considerably different amount of MAI in both cases.

In Fig. 5, the performance of adaptive uplink MC-CDMA is shown for the cases when CSI is available only at the transmitter and when CSI is available at both transmitter and receiver. In the case when CSI is available only at the transmitter, a pre-equalization technique which maximizes SINR is applied [3]. Adaptive MC-CDMA that exploits CSI at both transmitter and receiver employs G-pre-eq together with soft PIC (3 iterations). G-pre-eq with $p=1$ and $p=6$ is used in the case of full- and half-load, respectively. As references, the classical MF bound and the SD combined-equalization bound are given. In addition, performance results of uplink MC-CDMA with post-equalization applying soft PIC with 3 iterations (i.e., of uplink MC-CDMA without adaptivity) are shown. From Fig. 5 can be seen that adaptive uplink MC-CDMA 
Adaptivity in MC-CDMA Systems

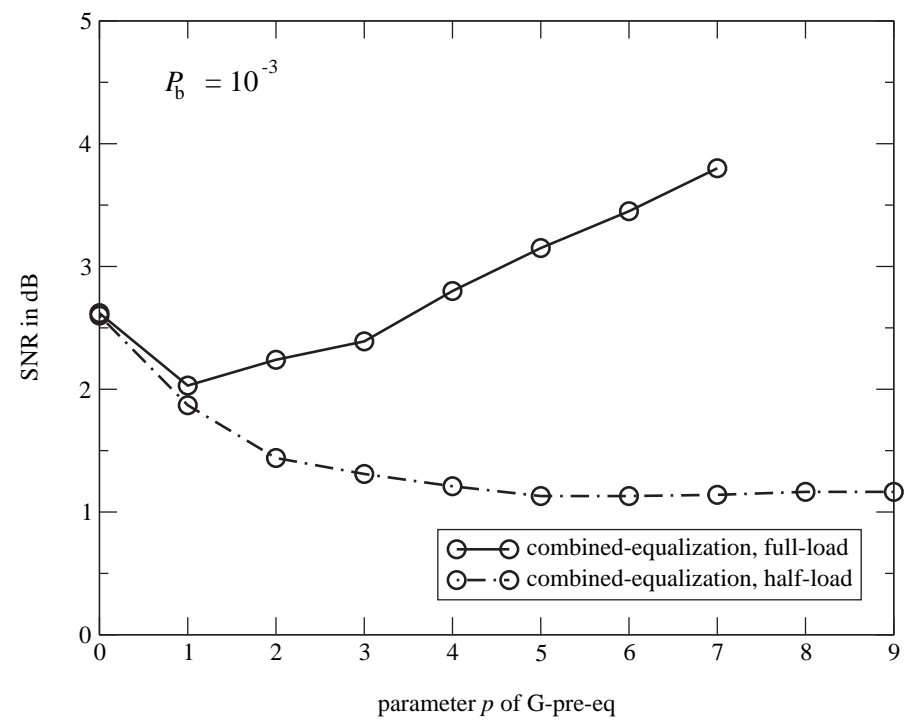

Figure 4. SNR required to achieve an average BER of $P_{\mathrm{b}}=10^{-3}$ versus the G-preeq parameter $p$ for adaptive uplink MC-CDMA with combined-equalization.

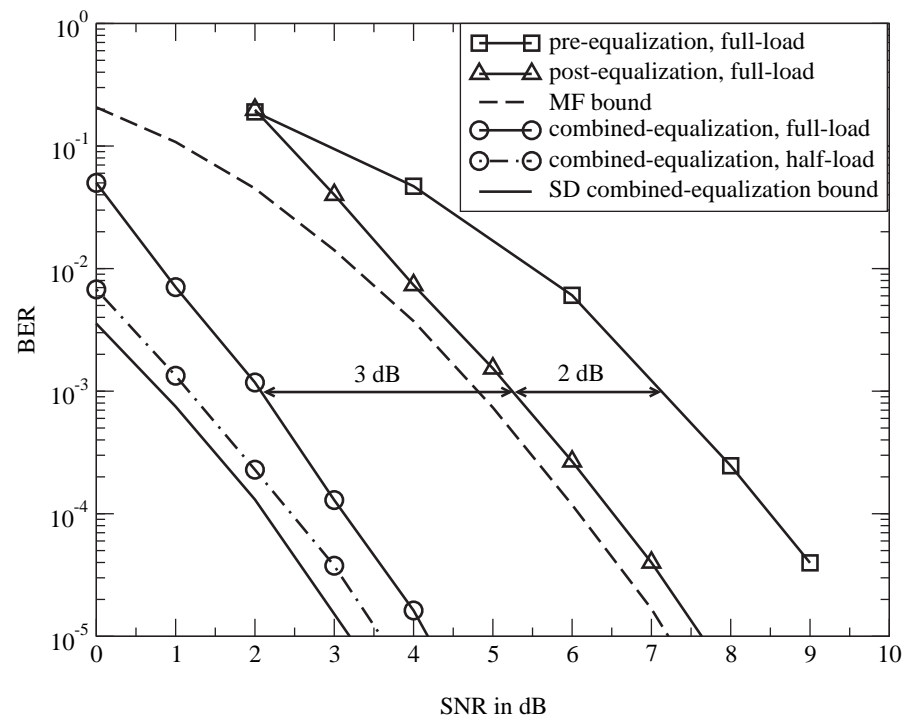

Figure 5. BER performance versus SNR for adaptive uplink MC-CDMA with preequalization and with combined-equalization.

that employs only pre-equalization performs worse than other considered techniques and is far from the MF bound. However, adaptive uplink MC-CDMA that employs combined-equalization outperforms others by several $\mathrm{dB}$ and approaches the SD combined-equalization bound closely.

The effects of imperfect CSI at the uplink transmitter on the system performance are considered in Fig. 6. We estimate CSI at the mobile 


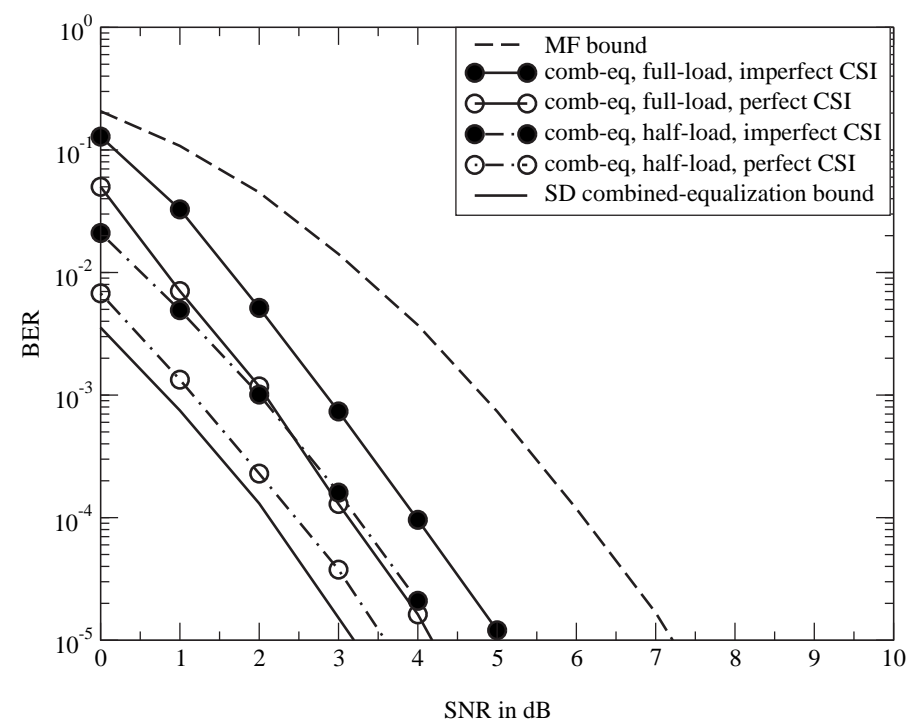

Figure 6. Effect of imperfect CSI at the mobile terminals on the BER performance of adaptive uplink MC-CDMA with combined-equalization.

terminal using a downlink channel estimation approach discussed in [7]. We exploit the estimates obtained from the pilot symbol inserted at the end of the downlink OFDM frame assuming that the power of this pilot symbol is boosted four times providing a more accurate CSI for the purposes of pre-equalization. We see that imperfect CSI induces an SNR loss of less than $1 \mathrm{~dB}$ irrespective of system load.

The impact of channel variations on the system performance is addressed in Fig. 7 where the SNR required to achieve a BER of $P_{\mathrm{b}}=10^{-3}$ is shown as a function of the mobile speed $v$. For pre- and combinedequalization the pre-equalization coefficients are computed at the beginning of each uplink frame and are kept fixed over the entire frame while the channel varies continuously according to the mobile speed $v$. As we can see, due to the soft PIC at the receiver, post- and combinedequalization are much more resistent to channel variations than preequalization. As expected the required SNR grows as $v$ increases, but the performance loss is relatively low for the mobile speeds up to $v \leq 5 \mathrm{~m} / \mathrm{s}$. Thus, the proposed scheme is well-suited for scenarios with low-mobility.

Finally, we address the effect of pre-equalization on the PAPR. Preequalization leads to power re-distribution at the transmitter. As shown in [4], the distribution of the transmission power strongly depends on the G-pre-eq parameter $p$. As $p$ grows, more power is invested on subcarriers with high gains and less on others. Already for $p=3$ more than $90 \%$ of the transmission power is concentrated on around $25 \%$ of available subcarriers. Considering the time domain representation most of the 


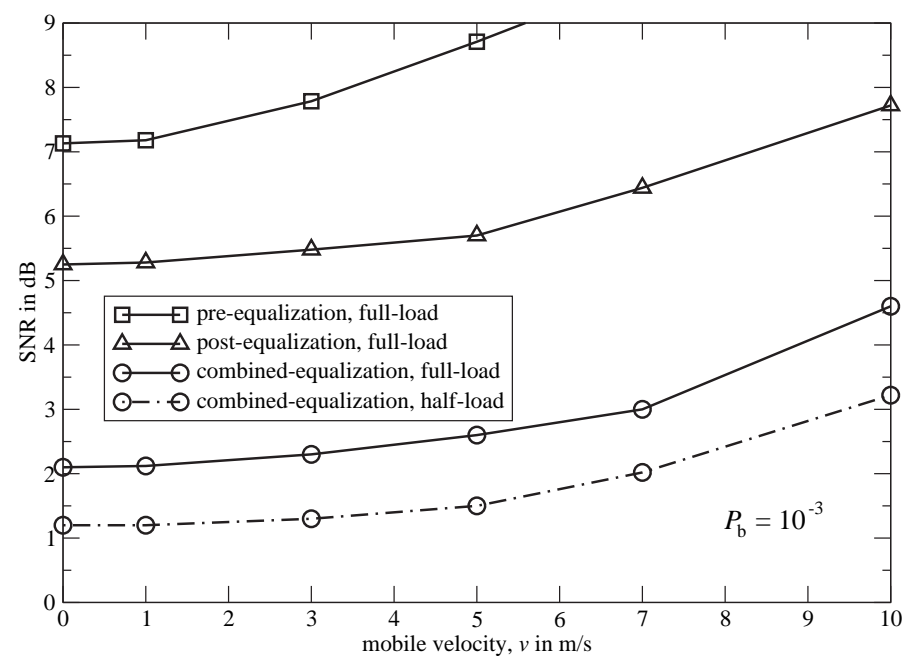

Figure 7. SNR required to achieve a BER of $P_{\mathrm{b}}=10^{-3}$ versus mobile speed $v$ for uplink MC-CDMA with combined-equalization, post-equalization, or pre-equalization.

power is concentrated on only a part of available sinusoidal waveforms. Such a situation leads to a lower PAPR, since there are fewer possibilities for constructive addition of signals. This is illustrated in Fig. 8 where a cumulative distributive function $(\mathrm{CDF})$ of the PAPR for uplink MC-CDMA applying different pre-equalization techniques is shown assuming, for simplicity reasons, that a system employs $Q$-modification. It can be seen that already for $p=3$ the PAPR is significantly reduced in comparison to pre-eq EGT. However, as shown in Fig. 4 adjustment of this parameter towards lower PAPR can lead to a worse BER performance, which can be partly compensated by introducing additional complexity at the base station, e.g., by introducing successive interference cancellation instead of PIC. Thus, in realistic uplink MC-CDMA with combined equalization, a certain trade-off between PAPR reduction, receiver complexity, and achievable performance can be sought.

\section{Conclusions}

Different approaches to exploit link adaptation in MC-CDMA are investigated for the uplink. Under consideration are pre-equalization and combined-equalization. As reference, post-equalization is also included. It could be shown that gains of $3 \mathrm{~dB}$ in SNR could be achieved when applying link adaptation compared to MC-CDMA systems without link adaptation. The presented results include the theoretical lower bounds and the performance of full and half loaded systems. Channel fading as well as real channel estimation is taken into account. Since the focus is on the uplink, the influence of different link adaptation approaches on 


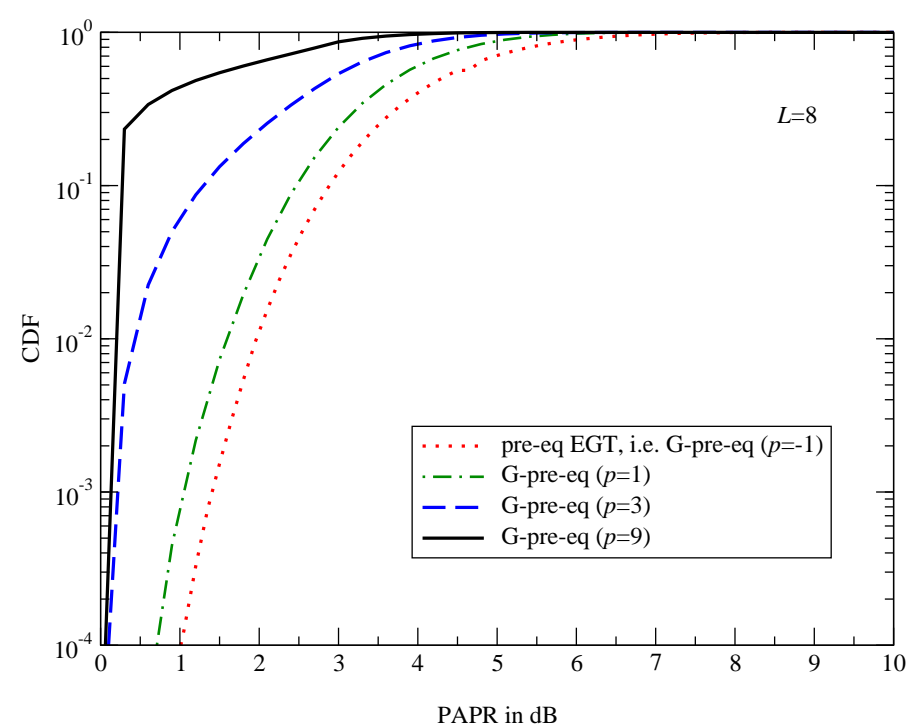

Figure 8. $\mathrm{CDF}$ for PAPR in $\mathrm{dB}$ for uplink MC-CDMA with pre-equalization for different G-pre-eq.

the PAPR is investigated and it could be shown that there is a trade-off between performance with link adaptation and reduction in PAPR.

\section{References}

[1] K. Fazel and S. Kaiser. Multi-Carrier and Spread Spectrum Systems. John Wiley \& Sons, 2003.

[2] C. Y. Wong, R. S. Cheng, K. B. Letaief, and R. D. Murch. Multiuser OFDM with adaptive subcarrier, bit, and power allocation. In IEEE J. Select. Areas Commun., pages 1747-1758, Oct. 1999.

[3] D. Mottier and D. Castelain. SINR-based channel pre-equalization for uplink multi-carrier CDMA systems. In Proceedings IEEE International Symposium on Personal, Indoor and Mobile Radio Communications (PIMRC'02), pages 14881492, Sept. 2002.

[4] I. Cosovic and S. Kaiser. Exploitation of diversity in MC-CDMA systems. In Proceedings IEE International Conference on 3G and Beyond (3G'05), Nov. 2005.

[5] S. Kaiser and J. Hagenauer. Multi-carrier CDMA with iterative decoding and soft-interference cancellation. In Proceedings IEEE Global Telecommunications Conference (GLOBECOM'97), pages 6-10, Nov. 1997.

[6] J. Medbo. Channel models for HIPERLAN/2 in different indoor scenarios. In ETSI EP BRAN 3ERI085B, March 1998.

[7] L. Sanguinetti, M. Morelli, and U. Mengali. Channel estimation and tracking for MC-CDMA signals. In European Transactions on Communications (ETT), pages 249-258, May/June 2004. 